\title{
The body schema: neural simulation for covert and overt actions of embodied cognitive agents
}

Pietro Morasso ${ }^{1 *}$ and Vishwanathan Mohan ${ }^{2}$

1 Department of Robotics, Brain and Cognitive Sciences, Istituto Italiano di Tecnologia, Via Enrico Melen 83, 16152, Genoa, Italy pietro.morasso@iit.it

2 School of Computer Science and Electronic Engineering, University of Essex Wivenhoe Park, CO34SQ, UK vishwanathan.mohan@essex.ac.uk

${ }^{*}$ Corresponding author

E-mail address: pietro.morasso@iit.it

Full postal address: Department of Robotics, Brain and Cognitive Sciences Istituto Italiano di Tecnologia Via Enrico Melen 83, 16152, Genoa, Italy

Keywords: Body schema, Proprioception, Embodied cognition, Neural simulation, Cognitive robotics 


\begin{abstract}
This brief commentary on the general topic of "body schema" is focused on its computational role, as an internal model that integrates proprioceptive information, for allowing embodied cognitive agents to carry out the neural simulation of covert and overt actions in a unitary manner. The discussion takes inspiration from the vintage but still valid seminal observation by Marr and Poggio that, in order to understand cognitive agents, both human and artificial, we should consider them as Generalized Information Processing Systems, to be analyzed along three levels: computational, algorithmic, and implementation. Accordingly, the body schema concept is briefly analyzed along this line, with the purpose of outlining a cognitive architecture that links embodied cognition with motor control through the body schema.
\end{abstract}




\section{Introduction: the body schema as a generalized information processing system}

The hypothesis that human purposive behavior requires some form of body representation, based on proprioceptive information, has been around for more than a century [1], supported by clinical observations that also suggested a multiplicity of representations [2-4]: Body Schema proper, Body Image, and Superficial Schema. Although such descriptive multiplicity is associated with a variety of clinical findings, we suggest that it highlights the multiple facets of a complex but unitary computational process: a somatotopically organized, extended body schema model, whose main function is the neural simulation of both real (overt) or imagined (covert) actions [5]. In this framework, such an extended body schema concept plays the crucial role of a bridge between embodied cognition [6] and motor control, enabling goal-directed actions guided by prospection $[7,8]$.

In general, cognitive agents - human or artificial - are characterized by the persistent need to link purposive actions to the prediction of their outcomes [9], a feature that was originally captured by Norbert Wiener with the invention of Cybernetics [10]. Such legacy was unfolded over the years and is still running through the contemporary developments of robotics and artificial intelligence, on one side, and neuroscience/cognitive science, on the other. In both cases, we can take inspiration from a seminal observation by Marr and Poggio [11] that, in order to understand cognitive agents, we should regard them as Generalized Information Processing Systems. This means, in particular, to articulate our investigation into three nearly independent but complementary levels of analysis, avoiding a widespread tendency to mix them: 1 ) the computational level that should clarify what needs to be computed and why; 2 ) the algorithmic level, focused on how the computation is carried out, in terms of the used representations and the processes employed to build and manipulate the representations; 3 ) the implementational/physical level, related to the selection and activation of the specific neural hardware used to carry out the computation. Although this epistemic approach was applied mostly to vision, there is ground to believe that the view of David Marr included motor cognition as well, considering his early work on theories of cerebellum, hippocampus, and neocortex: probably, he simply did not have time to do it for his premature death.

\section{The computational level}

A computational theory of the body schema may stem from the fact that a cognitive agent is continually involved in prospectively-guided, goal-directed actions and thus is faced by the challenge of choosing an action course on the basis of the predictable outcome. The literature on motor imagery, with particular emphasis on the studies related to the body schema [12-14], has clearly shown that overt actions are just the tip of an iceberg because, 'under the surface', is hidden a vast territory of covert counterparts, i.e. actions 'without movement'. The purpose of covert actions is to predict the likely outcome of a course of action, for understanding and deciding: for example, to restrain from acting, if the predicted result is dangerous and/or clearly leading to failure, or to analyze the anticipated failure for obtaining hints about a change of plan, and so on [15]. Consequently, the body schema should not be conceived as a passive and static representation of the body but as an active and dynamic mechanism, including the plastic extension to the skilled usage of tools [16] recruited and animated in a taskoriented manner. At this level of analysis, it is also necessary to consider the vexing question about the highdegree of redundancy of the human body that forces the cognitive/control system to choose action synergies among an infinite number of solutions: consider, in particular, Bernstein's Degrees of Freedom problem [17] and Lashley's Principle of Motor equivalence [18]. 
Shortly, what needs to be computed by the body schema, as a generalized information processing system driven by proprioceptive information, is a detailed prediction of the possible outcome of whole-body actions, generated according to a combination of goals and task constraints. Such predictions, while being fundamental for the skilled behavior of cognitive agents, also play a role in anticipating others' actions/goals, a capability that is crucial for imitation and skill transfer.

\section{The algorithmic level}

For the algorithmic level of analysis of the body schema, we suggest to focus on the simulation and emulation theories for the representation of prospectively-guided, goal-directed actions. Emulation theory $[19,20]$ assumes that an abstract representation of movement kinematics is maintained internally over short periods of time, in such a way to support motor imagery, both self-initiated or externally triggered: an emulator typically receives input from motor planning and outputs the sensory consequences as though the movement has been executed. Simulation theory $[5,21]$ postulates that imagined actions rely on the same set of neural mechanisms as the real actions, hence encapsulating in this mechanism the goal, the motor plan and sensory consequences. For example, observing someone "drinking a cup of coffee" or paying attention to the sentence "she drinks a cup of coffee" entails a simulation recruiting brain structures that overlap with the structures involved when one actually" drinks a cup of coffee" [22].

Internal emulation or simulation mechanisms, that were also advocated in cognitive robotics [23], may be interpreted as dynamic processes that animate the body schema. However, what still needs to be clarified is how the internal simulation may actually be organized and carried out. One possibility is that the animation of covert actions is carried out by using an internal model separate and independent of the model used for overt actions [24-26]. The alternative approach is based on the idea that the internal simulation of covert actions is performed by the same neural mechanism normally involved in overt actions, but relying on endogenous rather than exogenous sensorimotor signals $[15,27,28]$. The latter approach is certainly more parsimonious than the former one and is supported by a large body of direct and indirect evidence; moreover, it is linked to the still influential tradition underlying the ideomotor theories of the $19^{\text {th }}$ century $[29,30]$, namely the concept that the 'idea' of an action, i.e. the predicted/desired sensory consequences of a covert action, applies as well to goal-directed overt actions.

The question is then to figure out an algorithmic formulation of the 'idea' of the ideomotor theory that can tackle the redundancy of the motor system, without being stuck by the need to directly solve the inverse kinematic problem, i.e. choosing one among the infinite number of joint rotation patterns that are compatible with a desired trajectory of the end-effector. The proposed solution is a generalization of the Equilibrium-Point Hypothesis (EPH), introduced in the 1960's and debated ever since [31-33]. The rationale of the EPH is that the central nervous system generates overt actions as a shift of the equilibrium posture for both single- and multijoint movements, exploiting the spring-like properties of the neuromuscular system. The generalization of EPH, for including both covert and overt actions, was carried out by defining the Passive Motion Paradigm (PMP) [3435]. The basic idea, along with Jeannerod's simulation theory, is that overt and covert actions are both generated by a simulation process that "animates" the neural body schema. The novelty of PMP is to explain how the animation may be carried out: in simple terms, this neural process can be assimilated to the animation of a 'passive' marionette (the body schema) by means of a few wires (the virtual forces), pulled by the puppeteer, according to a storyboard (the task plan). Remarkably, this force-based mechanism solves the "Degrees of Freedom problem" in an implicit manner, without explicit kinematic inversion, and it naturally allows to combine 
multiple goals by superimposing the corresponding force fields. It is worth pointing at the analogy between PMP and Active Inference [36-37]: in both cases, there is no need to have distinct sensory and motor representations, because the 'proprioceptive predictions' of the intended action, generated by the simulation process, are sufficient to allow the motor controller to produce the basic motor synergies. Such predictions encode beliefs about the state of the world, including both proprioceptive and exteroceptive components. The standard causality between sensory and motor representations is somehow inverted: motor commands are not necessarily intended to cause desired movements - but desired movements may cause motor commands (in the form of the predicted consequences of movement). Moreover, the body schema framework also allows an agent to address the imitation game with a master, by providing the machinery for simulating the exemplary actions of the master and anticipate his intentions.

Considering that the algorithmic level is supposed to focus on how the computation is carried out, we may summarize the discussion above by saying that whole-body, goal-oriented synergies, for both overt or covert actions, may be generated by an animation process of the body schema, based on the PMP.

\section{The implementation/physical level}

The algorithmic hypothesis, that the same body schema is involved in the generation of overt and covert actions, can lead to different implementation strategies for transforming a selected covert action into the corresponding overt counterpart. One possibility is that the proprioceptive predictions of a planned action are used as reference signals by simple feedback control mechanisms, one for each DoF: such servomechanisms would attempt to minimize the difference between the proprioceptive predictions and the measured sensory feedback signals. This is an implementation strategy that is frequently used in robotics, relying on the fact that robotic technologies allow to provide sensory feedback signals that are virtually noiseless and delay-less. In human physiology this is certainly not the case and the real-time control of overt actions is implemented by a combination of different control strategies (feedforward, feedback, and stiffness control), tailored to specific task and environmental conditions and aimed at compensating noise and delay of sensory signals.

The crucial point is that synergy formation (of covert actions) and motor control (of overt actions), although interrelated and deeply complementary, are logically independent processes, with different computational mechanisms, different constraints, and different timelines. Synergy formation prescribes the recruitment of different groups of joints and muscles and the basic timing, reflected in the spatio-temporal invariances of biological motion [38], but is not and cannot be concerned with the biomechanics of the body, the dynamics of the environment, or haptic interaction; moreover, a great part of the covert actions remain unnoticed, inside the virtual domain of mental simulations.

Consider, for example, the act of whole-body reaching while standing $[39,40]$ : this an example of a dual task, with a 'focal subtask' (reach the designated target with the hand) and a 'postural subtask' (keep the projection of the center of mass - CoM - inside the basis of support - BoS). For simplicity, let us focus on a planar paradigm, related to reaching movements in the sagittal plane: the task is bidimensional, with a constraint about the horizontal position of the CoM, and there are 6 available DoFs (ankle, knee, hip, shoulder, elbow, wrist). Thus, this is a redundant task, with infinite solutions and a potential interference between the two subtasks: focusing only on the former may cause the latter to be violated. The interference problem can be avoided by a task manager by applying the PMP to the body schema with two virtual force fields: a force applied to the hand that pulls it to the target and a force applied to the CoM for pushing the CoM projection away from the border of the 
BoS. By learning and remembering how to tune the two force fields, the task manager may induce the body schema to generate a stable whole-body reaching synergy, in the form of a 6-dimensional proprioceptive prediction; the crucial point is that, although such a multi-joint synergy may be statically stable, it is likely to be insufficient to activate all the involved muscles in such a way to provide dynamic stability of the body, i.e. avoiding a fall. This goal is outside the competence of the synergy formation mechanism and can be achieved by the motor controller by combining three control strategies and recruiting them simultaneously: 1) A feedforward strategy, namely a bias of the antigravity muscles, driven by the proprioceptive prediction above; 2) A stiffness strategy, consisting of the coactivation of antagonist muscles for achieving joint stiffness values sufficient to compensate for unaccounted internal and external disturbances; 3) A feedback strategy, driven by delayed sensory information of body sway, for consolidating the overall dynamic stability of the action.

The first control action provides the basic skeleton of muscle recruitment and activation, satisfying the static criterion of stability. The second one is simple and functional for most joints of the kinematic chain, but is certainly ineffective for the ankle joint, which is critical for the dynamic stability of the standing body: measured values of ankle stiffness are indeed smaller than the crucial rate of growth of the gravity toppling torque due to body sway [41]. As a consequence, the third control action, limited to the ankle joint, is crucial. The main problem to be faced is that the feedback signal about the ongoing sway is delayed by an amount that is comparable to the natural falling time constant of the body 'inverted pendulum': the risk is that a continuous delayed feedback control could enhance rather than eliminate the danger of falling. The solution adopted by the motor controller is an intermittent control policy that alternates off-phases and on-phases during postural oscillations [42-44]: active feedback control is switched on if the state of the inverted pendulum (tilt angle and angular velocity) is departing from the equilibrium condition, whereas it is switched off in the opposite case. In this manner, dynamic stability of the standing body is not a rigid equilibrium point but a robust, persistent postural oscillation (sway).

We would like to stress the separation but also the deep complementarity between body-schema driven synergy formation, on one side, and the body-environment interaction, including the specific control action, on the other [45]: for example, intermittency characterizes the latter but not the former computational process. Synergy formation, that operates on the body schema distributing the action to the redundant set of degrees of freedom of the body, incorporates the constraint of static equilibrium in the spatio-temporal patterns of proprioceptive predictions; however, the actual dynamic stabilization of the initial and final postures is carried out by a hybrid combination of different mechanisms that exploit muscle stiffness and intermittent feedback control.

In summary, the implementation/physical level of analysis of the body schema concept is mainly concerned with the recruitment and tuning of different control mechanisms, that may allow the detailed proprioceptive predictions to meet the challenge of the interaction of the body with the physics of the real world.

\section{Conclusion}

This commentary analyzed the body schema as a Generalized Information Processing System, highlighting the fact that it is at the center of a cognitive architecture that supervises and coordinates overt and covert actions for skilled behavior. Figure 1 summarizes the separation but deep complementarity between the two main blocks of the cognitive architecture, namely the block that carries out muscle-less synergy formation, mainly in charge of covert actions, and the motor controller, responsible of the actual generation of muscle synergies. What flows from the cognitive to the control block is a continuous pattern of sensory predictions, while a pattern of corresponding sensory feedback signals flows in the opposite direction. Both patterns are multimodal, including 
in particular proprioceptive, tactile, and visual components [47-50], with a predominance of the proprioceptive component. Although the main function of this bidirectional flow of sensory information is related to the controlcognitive framework, another critical function, from the implementation point of view, is related to the need of calibrating and maintaining/updating the calibration of the body schema. This problem is central in the robotic field because multisensory calibration is essential for effective performance of cognitive robots $[51,53]$. Finally, we need to contrast this view of the body schema, as an information processing system for embodied cognition, to radically different formulations like the Smart Vehicles of Valentino Braitenberg [54], the refusal of neural representations by Rodney Brooks [55] or the reduction of cognition to agent-environmental dynamics by Anthony P. Chermero [56]. In particular, let us briefly focus on the criticism of Brooks, who advocates a totally bottom-up architecture that is supposed to achieve "intelligence without representation": this architecture is organized in layers, decomposing complicated intelligent behaviors into many "simple" behavioral modules, which in turn are organized into layers of simpler behaviors, down to reflex-like reactive mechanisms. Generally, the main problem of layered bottom-up architectures is that they scale-up badly when one attempts to deal with complex bodies and complex behaviors in a complex environment. Moreover, intelligence without representation may capture smart skills but is completely unable of prospective behavior that is based on the systematic and cumulatively use of action memory in its different forms: working, procedural, and episodic $[9,57]$. 


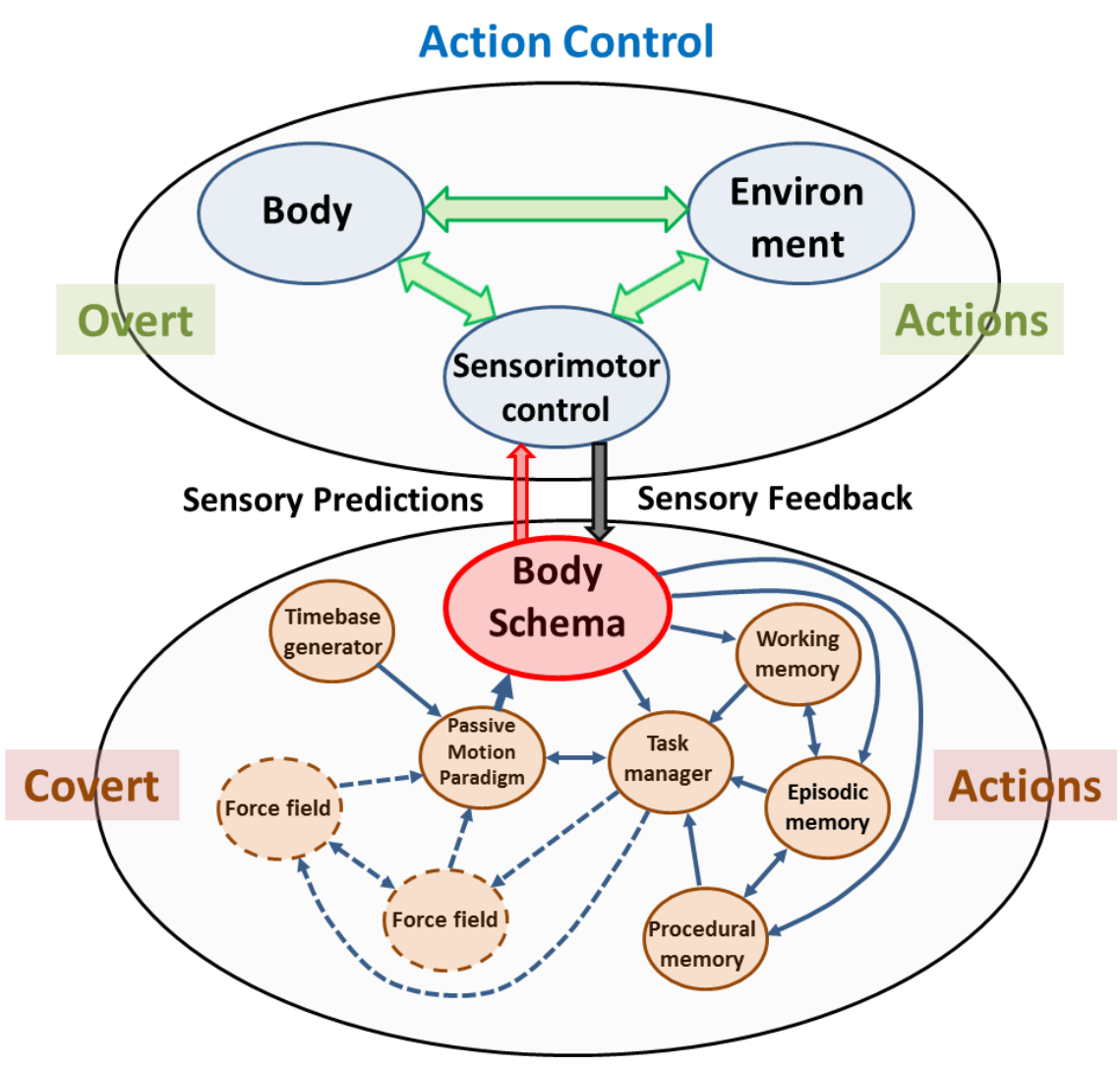

\section{Embodied Cognition}

Figure 1. Schematic diagram of a cognitive architecture built around the body schema model as a Generalized Information Processing System. The body schema is a somatotopic structure, holding a proprioceptive image of the body, that is animated by the Passive Motion Paradigm (PMP), thus generating a flow of sensory predictions related to goal-oriented covert actions. Most of them remain hidden, thus carrying out the fundamental prospective function of an embodied cognitive system, namely foresight and anticipation of future consequences of an action, including assessment and re-planning. The selected action plans are transformed by the sensorimotor control system, combining the appropriate control strategies, as a function of biomechanics and physics of the environment. The animation of the PMP is organized by the Task Manager that instantiates task-related Force Fields, recalling and exploiting memory traces of previous experiences accumulated during training and practice. 


\section{References and recommended reading}

Papers of particular interest, published within the period of review, have been highlighted as:

- of special interest

$\bullet \bullet$ of outstanding interest

1. Head H, Holmes G: Sensory disturbances from cerebral lesions. Brain 1911, 34:102-254 https://doi.org/10.1093/brain/34.2-3.102.

2. Schwoebel J, Coslett HB: Evidence for Multiple, Distinct Representations of the Human Body. Journal of Cognitive Neuroscience 2005, 17(4): 543-553 https://doi.org/10.1162/0898929053467587.

3. Pitron V, de Vignemont F: Beyond differences between the body schema and the body image:insights from body hallucinations. Consciousness and Cognition 2017, 53:115-121 https://doi.org/10.1016/j.concog.2017.06.006.

- $\quad$ By using the experimental evidence from hallucinations the authors attempt to answer the following questions: Are the body image and the body schema somehow reshaping each other or are they relatively independent and do they only happen to be congruent?

4. Pitron V, Alsmith A, de Vignemont F. (2018) How do the body schema and the body image interact? Consciousness and Cognition 2018, 65:352-358 https://doi.org/10.1016/i.concog.2018.08.007.

- A model is proposed for explaining how and why body schema and body image, as two complementary forms of representation, modify each other.

5. Jeannerod M: Neural simulation of action: a unifying mechanism for motor cognition. Neurolmage 2001, 14(1): S103-S109 https://doi.org/10.1006/nimg.2001.0832

6. Wilson M: Six Views of Embodied Cognition. Psychonomic Bulletin \& Review 2002, 9 (4): 625-636 https://doi.org/10.3758/bf03196322.

7. Maturana HR, Varela FJ: The tree of knowledge: The biological roots of human understanding. Boulder:Shambhala Pub; 1987: 0877736421.

8. von Hofsten C: An action perspective on motor development. Trends Cogn Sci 2004, 8: 266-272 https://doi.org/10.1016/j.tics.2004.04.002.

9. Vernon $D$, Beetz $M$, Sandini G: Prospection in cognitive robotics: The case for joint episodic-procedural memory. Frontiers in Robotics and Al 2015, 2: 19 https://doi.org/10.3389/frobt.2015.00019.

10. Wiener N: Cybernetics: Or Control and Communication in the Animal and the Machine. Cambridge:MIT Press; 1948: 9780262537841.

11. Marr D, Poggio T: From Understanding Computation to Understanding Neural Circuitry. MIT Libraries 1976, AIM-357: 1-21.

- This internal memo of the MIT Artificial Intelligence Lab did not have time to be reformulated as a full paper because David Marr died soon thereafter. However, it was and is still very influential after more than 40 years, having paved the road for a stricter collaboration between neuroscience/physiology, on one side, and cybernetics/artificial intelligence, on the other.

12. Schwoebel J, Boronat CB, Coslett HB: The man who executed "imagined" movements: Evidence for dissociable components of the body schema. Brain and Cognition 2002, 50: 1-16 https://doi.org/10.1016/S0278-2626(02)00005-2.

13. Shenton JT, Schwoebel J, Coslett HB: Mental motor imagery and the body schema: evidence for proprioceptive dominance. Neuroscience Letters 2004, 370: 19-24 https://doi.org/10.1016/j.neulet.2004.07.053. 
14. Baccarini M, Martel M, Cardinali L, Sillan O, Farnè A, Roy AC: Tool use imagery triggers tool incorporation in the body schema. Frontiers in Psychol 2014, 5: 492 https://doi.org/10.3389/fpsyg.2014.00492.

15. Mohan V, Bhat A, Morasso P: Muscleless Motor synergies and actions without movements: From Motor neuroscience to cognitive robotics. Physics of Life Reviews 2019, 30: 89-111 https://doi.org/10.1016/j.plrev.2018.04.005.

- This paper explains in details the rationale of this commentary.

16. Maravita A, Iriki A: Tools for the body (schema). Trends Cogn Sci 2004, 8: 79-86 https://doi.org/10.1016/j.tics.2003.12.008.

17. Lashley KS: Integrative function of the cerebral cortex. Physiological Reviews 1933, 13(1):1-42 https://doi.org/10.1152/physrev.1933.13.1.1.

18. Bernstein N: The co-ordination and regulation of movements. Oxford: Pergamon Press; 1967: B0007ITDZ8

19. Grush R: The emulation theory of representation: motor control, imagery, and perception. Behav Brain Sci 2004, 27(3): 377-396 https://doi.org/10.1017/s0140525x04000093.

20. Ptak R, Schnider A, Fellrath J: The dorsal frontoparietal network: a core system for emulated action. Trends Cogn Sci 2017, 21(8): 589-599 https://doi.org/10.1016/j.tics.2017.05.002.

- Starting from the evidence about the involvement of the dorsal frontoparietal network of the human brain in a variety of motor and cognitive functions (from motor planning and imagery to mental rotation, spatial attention, and working memory) it is proposed that this network can be understood as a system for the internal emulation of actions, with a generality of cognitive and motor functions.

21. O'Shea H, Moran A: Does motor simulation theory explain the cognitive mechanisms underlying motor imagery? A critical review. Front Hum Neurosci 2017, 11:72

https://doi.org/10.3389/fnhum.2017.00072.

- This review analyzes in depth the linkage between the motor simulation theory, formulated by Marc Jeannerod, and motor imagery, proposing that they share certain mental representations and mechanisms with action execution, hence activating similar neural pathways.

22. Jeannerod $M$ : The representing brain: neural correlates of motor intention and imagery. Behav. Brain Sci 1994, 17(2): 187-202; doi: https://doi.org/10.1017/S0140525X00034026.

23. Vernon D: Internal simulation in embodied cognitive systems. Phys Life Rev 2019, 30:122-125 https://doi.org/10.1016/i.plrev.2019.02.012.

- This commentary is a clear explanation of the need of cognitive agents (humans or cognitive robots) to continually predict the need for actions and their outcomes and the related emerging support of the concept that internal simulation plays a key role in such prospectively-guided goal-directed action.

24. Kawamura K, Gordon SM, Ratanaswasd P, Erdemir E, Hall JF: Implementation of cognitive control for a humanoid robot. Int J Humanoid Robot 2008, 5(4): 547-586

https://doi.org/10.1142/S0219843608001558.

25. Wolpert DM, Miall RC, Kawato M: Internal models in the cerebellum. Trends Cogn Sci 1998, 2(9): 338347 https://doi.org/10.1016/S1364-6613(98)01221-2.

26. Kunze L, Beetz M: Envisioning the qualitative effects of robot manipulation actions using simulationbased projections. Artif Intell 2017, 247:352-80 https://doi.org/10.1016/j.artint.2014.12.004.

- This paper focuses on envisioning, namely a qualitative reasoning method that reasons about actions and their effects based on simulation-based projections. These methods allow a robot to infer what could 
happen when it performs a task in a certain way but can also provide a rationale for understanding the organization of human behavior and the role of the body schema in this framework.

27. Shanahan MP: A cognitive architecture that combines internal simulation with a global workspace. Conscious Cogn 2006, 15(2): 433-449 https://doi.org/10.1016/j.concog.2005.11.005 .

28. Hesslow G: The current status of the simulation theory of cognition. Brain Res 2012, 1428: 71-79 https://doi.org/10.1016/j.brainres.2011.06.026.

29. James W: The principles of psychology. New York:Henry Holt and Co; 1890:978-1-60206-284-9.

30. Shin YK, Proctor RW, Capaldi EJ: A review of contemporary ideomotor theory. Psychological Bulletin 2010, 136(6): 943-974 https://doi.org/10.1037/a0020541.

31. Feldman AG: Functional tuning of the nervous system with control of movement or maintenance of a steady posture: II controllable parameters of the muscle. Biophysics 1966, 11: 565-578

32. Feldman AG: Once more on the equilibirium hypothesis (lambda model) for motor control. J Mot Behav 1986, 18: 17-54 https://doi.org/10.1080/00222895.1986.10735369.

33. Bizzi E, Hogan N, Mussa-Ivaldi FA, Gistzter S: Does the nervous system use equilibrium-point control to guide single and multiple joint movements? Behav and Brain Sciences 1992, 15(4): 603-613 https://doi.org/10.1017/S0140525X00072538.

34. Mussa Ivaldi FA, Morasso $P$, Zaccaria R: Kinematic networks. A distributed model for representing and regularizing motor redundancy. Biol Cybern 1988, 60: 1-16 https://doi.org/10.1007/BF00205967.

35. Mohan V, Morasso P: Passive Motion Paradigm: an alternative to Optimal Control. Frontiers in Neurorobotics 2011, 5: 4 https://doi.org10.3389/fnbot.2011.00004.

36. Friston K, Mattout J, Kilner J: Action understanding and active inference. Biol Cybern 2011, 104: 137160 https://doi/10.1007/s00422-011-0424-z.

37. Friston KJ, ParrT: Passive motion and active inference. Phys Life Rev 2019, 30: 112-115 https://doi.org/10.1016/j.plrev.2019.01.004.

- This paper explains how two apparently different models, passive motion and active inference, are in fact complementary paradigms for understanding an embodied interaction between the body schema and the world.

38. Johansson G: Visual perception of biological motion and a model for its analysis. Perception \& Psychophysics 1973, 14(2): 201-211 https://doi.org/10.3758/BF03212378.

39. Morasso P, Casadio M, Mohan V, Zenzeri J: A neural mechanism of synergy formation for whole body reaching. Biol Cybern 2010, 102(1): 45-55 https://doi.org/10.1007/s00422-009-0349-y .

40. Morasso P, Casadio M, Mohan V, Rea F, Zenzeri J: Revisiting the body-schema concept in the context of Whole-Body Postural-Focal Dynamics. Frontiers Human Neurosci 2015, 9: 83 https://doi.org/10.3389/fnhum.2015.00083.

41. Casadio $M$, Morasso $P$, Sanguineti $V$ : Direct measurement of ankle stiffness during quiet standing: implications for control modelling and clinical application. Gait \& Posture 2005, 21: 410-414 https://doi.org/10.1016/j.gaitpost.2004.05.005

42. Bottaro A, Yasutake $Y$, Nomura $T$, Casadio $M$, Morasso $P$ : Bounded stability of the quiet standing posture: an intermittent control model. Hum Mov Sci 2008, 27(3): 473-495 https://doi.org/10.1016/j.humov.2007.11.005.

43. Asai Y, Tasaka Y, Nomura K, Nomura T, Casadio M, Morasso P: A model of postural control in quiet standing: robust compensation of delay-induced instability using intermittent activation of feedback control. PLoS One 2009, 4(7): e6169 https://doi.org/10.1371/journal.pone.0006169. 
44. Morasso P, Cherif A, Zenzeri J: Quiet Standing: the Single Inverted Pendulum Model is not so bad after all. PLoS One 2019, 14(3): e0213870 https://doi.org/10.1371/journal.pone.0213870.

45. Murata A, Maeda K, Naito E: Body schema as a link between motor control and cognitive function. Proc. IEEE ICME, July 1-4, 2012, Kobe, Japan, 467-470 https://doi.org/10.1109/ICCME.2012.6275708 .

46. Maravita A., Charles Spence C., Driver J: Multisensory Integration and the Body Schema: Close to Hand and Within Reach. Current Biol 2003, 13(13): R531-R539 https://doi.org/10.1016/S09609822(03)00449-4.

47. Cardinali L, Brozzoli C, Luauté J, Roy AC, Farnè A: Proprioception is necessary for body schema plasticity: evidence from a deafferente patient. Frontiers Human Neurosci, Case Reports 2016, 10: 272 https://doi.org/10.3389/fnhum.2016.00272.

- This study clearly demonstrates the crucial importance of proprioception for the body schema.

48. Giurgola S, Pisoni A, Maravita A, Vallar G, Bolognini N: Somatosensory cortical representation of the body size. Hum Brain Mapp 2019, 40: 3534-3547 https://doi.org/10.1002/hbm.24614 .

- This study demonstrates, by repetitive Transcranial Magnetic Stimulation to the somatosensory cortical map of the hand in both hemispheres, that perturbed proprioception determines a perceptual distortion of the hand size.

49. Isayama R, Vesia M, Jegatheeswaran G, Elahi B, Gunraj CA, Cardinali L, Farnè A, Chen R: Rubber hand illusion modulates the influences of somatosensory and parietal inputs to the motor cortex. J Neurophysiol 2019, 121(2): 563-573 https://doi.org/10.1152/jn.00345.2018.

- By using the transcranial magnetic stimulation, in the context of the rubber hand illusion paradigm, this study provides support to the concept that parietal-motor connection is involved in resolving sensory conflicts and body ownership during the illusory paradigm.

50. Sadibolova R, Ferrè ER, Linkenauger SA, Longo MR: Distortions of perceived volume and length of body parts. Cortex 2019, 111: 74-86 https://doi.org/10.1016/j.cortex.2018.10.016.

- Considering that distortions of the body image are central to several serious diseases, this study proposes a simple method for evaluating the distortion of volume and length perception in normal subjects, as a baseline for clinical studies.

51. Fitzpatrick P, Metta C: Grounding vision through experimental manipulation. Phil Trans Royal Soc $A$ 2003, 361(1811): 2165-2185 https://doi.org/10.1098/rsta.2003.1251.

52. Vicente $P$, Jamone $L$, Bernardino A: Online body schema adaptation based on Internal Mental Simulation and Multisensory feedback. Frontiers Robotics Al 2016, 3(7) https://doi.org/10.3389/frobt.2016.00007.

- This paper provides a novel approach to obtain automatic adaptation of the robot body schema and to improve the robot perceptual and motor skills based on this body knowledge.

53. Pugach G, Pitti A, Tolochko O, Gaussier P: Brain-inspired coding of robot body schema through visuomotor integration of touched events. Frontiers Neurorob 2019, 13(5) https://doi.org/10.3389/fnbot.2019.00005.

- Considering that Gain-Field neurons in the parietal cortex are known to be involved in computing the spatial transformations for multisensory fusion (aligning tactile, visual and proprioceptive signals) and the fact that in reaching tasks these neurons exploit a mechanism based on multiplicative interaction for binding simultaneous multimodal events, this work shows how to adapt the internal body schema of a robot by using such neural architecture, using an artificial skin. 
54. Braitenberg V: Vehicles: Experiments in synthetic psychology. Cambridge MA: MIT Press; 1984 ISBN 978-0-262-52112-3.

55. Brooks R: Intelligence without representation. Artif Intell J 1991, 47: 139-159 https://doi.org/10.1016/0004-3702(91)90053-M

56. Chemero AP: Radical Embodied Cognitive Science. Cambridge MA: MIT Press; 2009 ISBN: 9780262516471.

57. Mohan V, Sandini G, Morasso P: A neural framework for organization and flexible utilization of episodic memory in "cumulatively" learning baby humanoids. Neural Computation 2014, 26: 26922734 https://doi.org/10.1162/NECO a 00664.

\section{Competing interests statement}

The authors declare that they have no competing interests to declare.

\section{Acknowledgments}

This study was supported by RBCS (Robotics, Brain and Cognitive Science Department), Italian Institute of Technology, Genoa, Italy. 